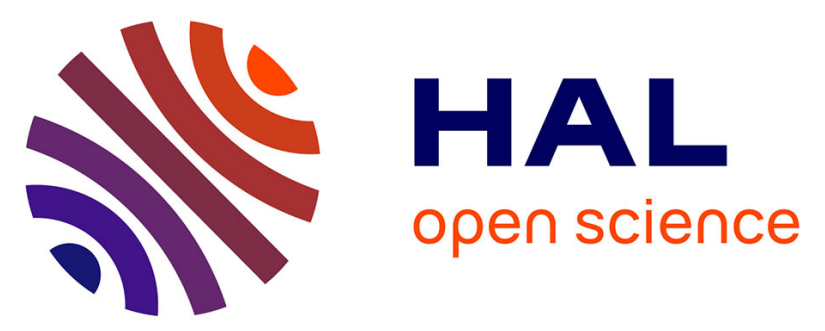

\title{
Environment-Aware RSSI Based Positioning Algorithm for Random Angle Interference Cancellation in Visible Light Positioning System
}

\author{
Yanqi Huang, Dayu Shi, Xun Zhang, El-Hassane Aglzim, Lina Shi
}

\section{- To cite this version:}

Yanqi Huang, Dayu Shi, Xun Zhang, El-Hassane Aglzim, Lina Shi. Environment-Aware RSSI Based Positioning Algorithm for Random Angle Interference Cancellation in Visible Light Positioning System. Indoor Positioning and Indoor Navigation, Nov 2021, Lloret de Mar, Spain. hal-03328942

\section{HAL Id: hal-03328942 \\ https://hal.science/hal-03328942}

Submitted on 31 Aug 2021

HAL is a multi-disciplinary open access archive for the deposit and dissemination of scientific research documents, whether they are published or not. The documents may come from teaching and research institutions in France or abroad, or from public or private research centers.
L'archive ouverte pluridisciplinaire HAL, est destinée au dépôt et à la diffusion de documents scientifiques de niveau recherche, publiés ou non, émanant des établissements d'enseignement et de recherche français ou étrangers, des laboratoires publics ou privés. 


\section{Environment-Aware RSSI Based Positioning Algorithm for Random Angle Interference Cancellation in Visible Light Positioning System}

\author{
$1^{\text {st }}$ Yanqi Huang \\ DRIVE EA1859, \\ Univ. Bourgogne Franche Comté, \\ F58000, Nevers France \\ Institut superieur d'electronique de Paris \\ Paris, France \\ Yanqi ${ }_{H} u a n g @ e t u . u-b o u r g o g n e . f r$ \\ $4^{\text {th }}$ El-Hassane Aglzim \\ DRIVE EA1859, Univ. Bourgogne Franche Comté, \\ F58000, Nevers France \\ el-hassane.aglzim@u-bourgogne.fr
}

$2^{\text {nd }}$ Dayu Shi

$3^{\text {rd }}$ Xun Zhang

Institut superieur d'electronique de Paris Institut superieur d'electronique de Paris

Paris, France

dayu.shi@ext.isep.fr
Paris, France

xun.zhang@isep.fr

\author{
$5^{\text {th }}$ Lina Shi \\ Laboratoire d'Ingénierie des Systèmes de Versailles, \\ Université de Versailles Saint-Quentin-en-Yvelines \\ Vélizy, France \\ linashi@lisv.uvsq.fr
}

\begin{abstract}
Visible Light Positioning (VLP) is considered to be one of the most promising candidates for future Location Based Service (LBSs). The traditional Received Signal Strength Indication (RSSI) based VLP system is highly sensitized with receiver's orientation. However, the assumption of the receiver's orientation fixed or perfectly known is not realistic in practice. Thus, a random angle between receiver and the horizontal plane inevitably appears among localization, which extremely affects positioning results. This paper proposed an Environment-Aware RSSI based positioning algorithm for VLP system. It enables to mainly eliminate random angle interference without the help of extra equipment. The positioning error caused by random angle was theoretically analyzed and validated by numerical simulation. Moreover, a demonstration on a real 5G New Radio (NR) signal platform was implemented to verify the feasibility of our algorithm. According to the results, the average positioning error declined by $\mathbf{7 3 . 2 8 \%}$.
\end{abstract}

Index Terms-Visible Light Positioning, Location Based Services, Received Signal Strength Indicator, Indoor Positioning System

\section{INTRODUCTION}

With the enormous growth of Internet of Things (IoT) applications and deep integration of information technology into a number of industrial scenarios, Location Based Services (LBS)s are urgently stimulated to be reformed from all dimensions. The familiar Global Positioning System (GPS) is now in everyday use around the world. Unfortunately, it is burdensome for the indoor environment due to the huge attenuation of satellite signal when passing through solid walls [1]. In order to fill the technical gap of indoor positioning, most approaches are based on the reception of radio frequency (RF) signals, e.g. WiFi [2], Bluetooth [3] or Ultra Wideband (UWB) [4]. However, the fundamental problem in radio based systems is multipath propagation. Radio signals may reach a receiver by both direct line of sight and multiple reflected paths. This means that there is no simple and reliable way of determining the distance or direction of the transmitter from the received signal [5].

Alternatively, Visible Light Positioning (VLP) is considered to be one of the most promising candidate for future LBS benefits f rom i ts s everal i rresistible a dvantages. F irstly, VLP system coexist with illumination, which reduce the complexity and cost of installation and maintenance. Meanwhile, LEDs as the transmitter provides VLP system with long lifetime, non electromagnetic radiation and low power consumption [6] [7]. Moreover, the position accuracy recorded in the literature is among the best of all indoor positioning approaches [8].

Most of VLP approaches relay on Received Signal Strength Indicator (RSSI) and strictly required the sensitive area of receiver paralleled with the ground to guarantee the accuracy. However, the assumption of the receiver's orientation fixed and known is not realistic in practice. Especially, when the receiver is held by users or integrated on robots during the movement, a random angle deviation between transmitter and receiver will be inevitably generated and significantly affects positioning results [9].

There existed several studies on the interference of receiver's orientation. E.-M. Jeong et al. presented a positioning approach with the help of an extra equipment to measure the orientation [10]. Q. Li et al. investigated an approach of nonlinear relationship model between RSS and the orientation 
of receiver. The prediction of the model and an iterative procedure based gradient descent algorithm were employed to fix positioning error [11]. D. Mai et al. proposed an enhanced positioning approach to minimize the positioning error by building up the Identification (ID) of LEDs in physical and link network layers [12]. In general, most of existed works required an extra equipment or extremely increased the complexity of system. Therefore, a random angle interference cancellation method that is high accuracy and dispenses with extra equipment is urgently required.

This paper proposed an Environment-Aware RSSI based positioning algorithm to eliminate the interference of random angle for VLP system. This proposal regards random angle interference as environment perturbations which can be described by a set of environment indexes. By establishing the mapping between them with received signals power beforehand, the deviation of random angle can be mainly eliminated from the positioning results. The main contributions of this paper are summarized as follows:

- The theoretical analysis and numerical simulation based validation indicated that the random angle interference is an important factor degrading the positioning accuracy.

- We proposed an Environment-Aware RSSI based positioning algorithm to eliminate the interference of random angle. The classification model is trained firstly in offline phase and is used in online phase to calculate coordinates of target.

- The proposed algorithm was validated by a series of numerical simulation and demonstrated on a realistic $5 \mathrm{G}$ New Radio (NR) signal based VLP platform. Demonstration results indicated that the proposed algorithm declined the average Positioning Error (PE) from $22.34 \mathrm{~cm}$ to $5.97 \mathrm{~cm}$.

The rest of this paper is organized as follows: Section II analyzes the traditional RSSI based VLP system and the positioning error caused by random angle interference. Section III details the framework of proposed algorithm. Section IV compares our proposed algorithm and traditional RSSI based VLP algorithm by a series of simulations. To validate the proposed algorithm, demonstration employed on a 5G NR signal based VLP platform is presented in Section V. Section VI concludes and discusses the advantages and the limitations of our proposal.

\section{TRADitional RSSI BASED VLP SYSTEM AND THE IMPACT OF RANDOM ANGLE INTERFERENCE}

In this section, the traditional RSSI based VLP system is introduced and the positioning deviation caused by random angle is derived theoretically.

In a typical VLP system, several LEDs are used as transmitters. The target is equipped with a Photodiode (PD) as a receiver. Traditional RSSI VLP system uses transmitterreceiver distances to calculate the estimated coordinate. Here, assumed that there are $N(N \geq 3)$ LEDs at the same height and the lights from LEDs are Line-of-Sight (LOS) propagation. To simplify illustration, LED $i, i \in[1, N]$ is taken as an example.
According to the characteristic of Optical Wireless Channel (OWC) [13], when receiver parallels with transmitter shown in Fig. 1 (a), incident angle $\Psi$ equals to radiation angle $\phi$. The calculation of distance $D_{i}$ between receiver and the LED $i$ can be derived by equation (1) (2):

$$
\Psi_{i}=\Phi_{i}
$$

$$
\begin{gathered}
D_{i}=\left(\frac{A_{P D}\left(m_{i}+1\right) \cdot P_{t}^{i} \cdot H^{m_{i}}}{2 \pi P_{r}^{i}} T_{s}\left(\Phi_{i}\right) g\left(\Phi_{i}\right) \cos \left(\Phi_{i}\right)\right)^{\frac{1}{m_{i}+2}} \\
m_{i}=\frac{-\ln 2}{\ln \cos \Phi_{1 / 2}^{i}}
\end{gathered}
$$

where, $H$ is vertical distance between LED $i$ and receiver, $A_{P D}$ is effective area of receiver. $P_{t}^{i}$ and $P_{r}^{i}$ are transmitted and received signal power of LED $i . T_{s}\left(\Psi_{i}\right)$ and $g\left(\Psi_{i}\right)$ are the gain of optical band-pass filter and non-imaging concentrator. The order of Lambertian emission $m_{i}$ for LED $i$ is defined as equation (3), where $\Phi_{1 / 2}^{i}$ is semi-angle at half-power of LED $i$.

As the Fig. 1(b)(c) show, when the receiver tilts a random angle $\theta_{i}$, the incident angle $\Psi_{i}$ is replaced by equation (4). Meanwhile the expression of distance $D_{i}^{\prime}$ is derived by equation (5). To describe the deviation caused by random angle, $\epsilon$ is defined and simplified as equations (6) (7) (8) (9).

$$
\begin{gathered}
\Psi_{i}=\Phi_{i}-\theta_{i} \\
D_{i}^{\prime}=\left(\frac{A_{P D}\left(m_{i}+1\right) \cdot P_{t}^{i} \cdot H^{m_{i}}}{2 \pi P_{r}^{i}} \cdot T_{s}\left(\Phi_{i}-\theta_{i}\right) g\left(\Phi_{i}-\theta_{i}\right)\right. \\
\left.\cdot \cos \left(\Phi_{i}-\theta_{i}\right)\right)^{\frac{1}{m_{i}+2}} \\
a_{i}=\frac{A_{P D} \cdot\left(m_{i}+1\right) \cdot P_{t}^{i} \cdot H^{m_{i}}}{2 \pi P_{r}^{i}} \\
f(\theta)=T_{s}\left(\Phi_{i}\right) g\left(\Phi_{i}\right) \cos \left(\Phi_{i}\right)-T_{s}\left(\Phi_{i}-\theta\right) g\left(\Phi_{i}-\theta\right) \cos \left(\Phi_{i}-\theta\right) \\
\epsilon=\sum_{i=1}^{N}\left(a_{i} \cdot\left(f\left(\theta_{i}\right)\right)\right)^{\frac{1}{m_{i}+2}} / N
\end{gathered}
$$

Therefore, the random angle will significantly affect the positioning accuracy. Our algorithm is proposed to eliminate this interference by declining the deviation $\epsilon$.

\section{ENVIRONMENT-AWARE RSSI BASED POSITIONING ALGORITHM}

The proposed algorithm regards the interference of random angle as environmental perturbations. The framework of the proposed algorithm is depicted in Fig. 2, which contains two phases: Offline (Classification model establishment) and online (Positioning). 


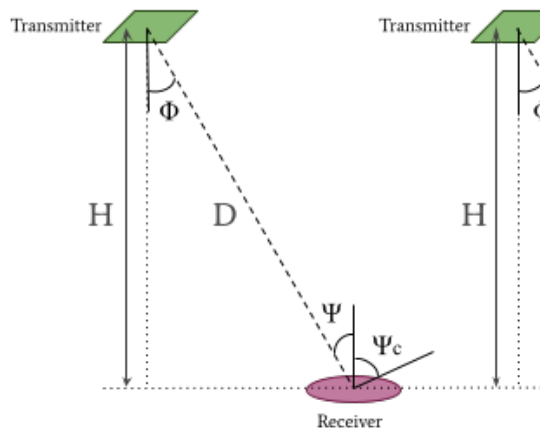

(a)

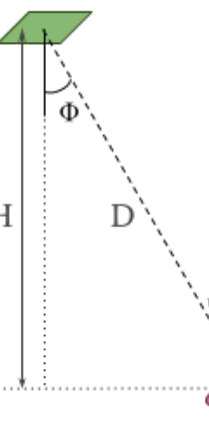

(b)

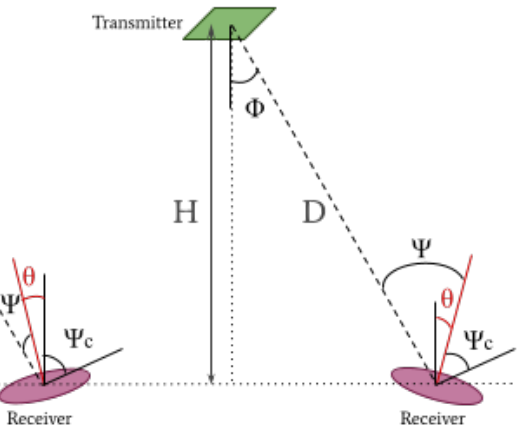

(c)

Fig. 1. Three possibilities of receiver attitude: (a) the receiver is parallel to the ground, (b) the angle of the receiver towards the LED is $\theta$, and (c) the angle of the receiver away from the LED is $\theta$.

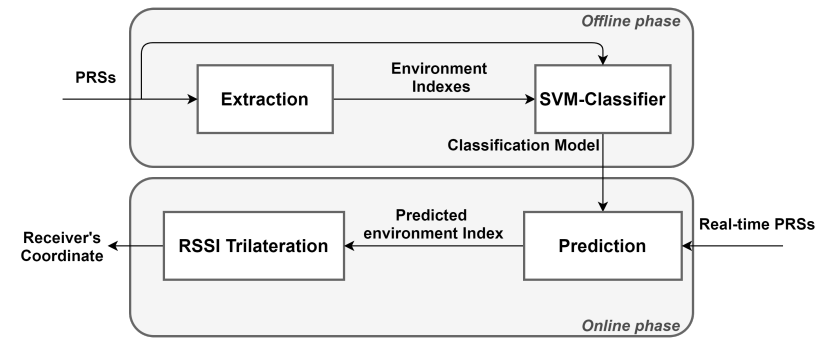

Fig. 2. The framework of Environment-Aware RSSI positioning algorithm

\section{A. The offline phase: Classification model establishment}

In this phase, a classification model $M$ was constructed to predict the environment index. Firstly, the extraction of environment indexes from positioning reference signals PRSs is employed. To simplify the illustration, the process of environment index $\lambda_{x y}$ extraction on $y t h(y \in[1, K])$ reference point for $x t h(x \in[1, N])$ LED is detailed as an example. The estimated distance $\left(\hat{D_{x y}}\right)$ was calculated by received PRSs power. During the signals collection, receiver tilts a random angle. Based on $n$ pairs of estimated and realistic distances $\left(D_{x y}\right)$, an optimization can be used to fit the environment index $\lambda_{x y}$ shown by equation (10) (11). The environment index $\lambda$ consists of two parameters (equation (12)), where $m$ and $G$ represent the compensation of Lambertian distribution and extra pathloss caused by random angle interference.

$$
\begin{gathered}
\hat{D}_{x y}=G_{x y}\left(\frac{\left(m+m_{x y}+1\right) A_{P D} P_{t} H^{\left(m+m_{x y}+1\right)}}{2 \pi P_{r}}\right)^{\frac{1}{m+m_{x y}+3}} \\
\min _{\lambda_{x y}} \sum_{i=1}^{n}\left[D_{x y}-\hat{D_{x y}}\left(P_{r}\right)\right] \\
\lambda=\{m, G\}
\end{gathered}
$$

Once environment indexes of all LEDs on all reference points have been extracted completely, the sets $\Lambda$ and $S$ (equations (13) (14)) are obtained and applied to train the classification model. This model builds up the mapping between environment indexes and the PRSs power shown in (15). In this paper, an one-against-all Multi-SVM classifier [14] has been employed.

$$
\begin{gathered}
\Lambda=\left\{\begin{array}{cccc}
\lambda_{11} & \lambda_{12} & \ldots & \lambda_{1 K} \\
\lambda_{21} & \ldots & \ldots & \\
\ldots & \ldots & \ldots & \\
\lambda_{N 1} & \ldots & \ldots & \lambda_{N K}
\end{array}\right\} \\
S=\left\{\begin{array}{cccc}
P_{11} & P_{12} & \ldots & P_{1 K} \\
P_{21} & \ldots & \ldots & \\
\ldots & \ldots & \ldots & \\
P_{N 1} & \ldots & \ldots & P_{N K}
\end{array}\right\} \\
M: S \rightarrow \Lambda
\end{gathered}
$$

\section{B. The online phase: Positioning}

The objective of this phase is to estimate the coordinates of the target receiver. When the receiver captures real-time PRSs powers of each LED, the corresponding environment indexes $\hat{\lambda}$ will be predicted by the classification model $M$ shown in equation (16). As the distances between receiver and each LED have been figured out, the set of distances $\hat{D}$ is used to estimate the coordinate of the receiver $(\hat{x}, \hat{y}, \hat{z})$ by RSSI trilateration, shown in equations (17) to (23).

$$
M\left(\left[P_{r}^{1}, P_{r}^{2}, \ldots, P_{r}^{N}\right]\right) \rightarrow \hat{\lambda}(\hat{m}, \hat{G})
$$

$$
\left\{\hat{D} \mid \hat{D}_{i}=\left(\frac{\left(m_{0}+\hat{m}+1\right) A_{P D} P_{t}^{i} H^{\left(m_{0}-\hat{m}+1\right)}}{2 \pi P_{r}^{i}}\right)^{\frac{1}{m_{t}+3}}, i \in[1, N]\right\}
$$

$$
\left\{\begin{array}{c}
\left(x_{1}-\hat{x}\right)^{2}+\left(y_{1}-\hat{y}\right)^{2}+\left(z_{1}-\hat{z}\right)^{2}=\hat{D_{1}} \\
\left(x_{2}-\hat{x}\right)^{2}+\left(y_{2}-\hat{y}\right)^{2}+\left(z_{2}-\hat{z}\right)^{2}=\hat{D_{2}} \\
\vdots \\
\left(x_{N}-\hat{x}\right)^{2}+\left(y_{N}-\hat{y}\right)^{2}+\left(z_{N}-\hat{z}\right)^{2}=\hat{D_{N}}
\end{array}\right.
$$




$$
\begin{gathered}
A X=B \\
X=\left[\begin{array}{c}
\hat{x} \\
\hat{y} \\
\hat{z}
\end{array}\right] \\
A=\left[\begin{array}{ccc}
2 x_{2}-2 x_{1} & 2 y_{2}-2 y_{1} & 2 z_{2}-2 z_{1} \\
2 x_{3}-2 x_{1} & 2 y_{3}-2 y_{1} & 2 z_{3}-2 z_{1} \\
\vdots & \vdots & \vdots \\
2 x_{N}-2 x_{1} & 2 y_{N}-2 y_{1} & 2 z_{N}-2 z_{1}
\end{array}\right] \\
B=\left[\begin{array}{c}
D_{1}^{2}-D_{2}^{2}-x_{1}^{2}+x_{2}^{2}-y_{1}^{2}+y_{2}^{2}-z_{1}^{2}+z_{2}^{2} \\
D_{1}^{2}-D_{3}^{2}-x_{1}^{2}+x_{3}^{2}-y_{1}^{2}+y_{3}^{2}-z_{1}^{2}+z_{3}^{2} \\
\vdots \\
D_{1}^{2}-D_{N}^{2}-x_{1}^{2}+x_{N}^{2}-y_{1}^{2}+y_{N}^{2}-z_{1}^{2}+z_{N}^{2}
\end{array}\right] \\
{\left[\begin{array}{c}
\hat{x} \\
\hat{y} \\
\hat{z}
\end{array}\right]=\left(A^{T} A^{-1}\right) A^{T} B}
\end{gathered}
$$

where $\left(x_{i}, y_{i}, z_{i}\right)$ represents the coordinate of $i t h$ LED. To evaluate the positioning accuracy, the positioning error is defined as equation (24):

$$
P E=\sqrt{(\hat{x}-x)^{2}+(\hat{y}-y)^{2}+(\hat{z}-z)^{2}}
$$

\section{Simulation Results}

In this section, to verify the feasibility of our proposal, a series of numerical simulations had been employed. Firstly, the simulation set up was introduced, which included the virtual scenario description, simulation parameters and the methodology of the simulation design. Subsequently, the disparity of our proposal and traditional RSSI VLP system on deviation elimination was visualized. Finally, the performance of transitional RSSI Based VLP system and our proposal was compared and analyzed.

\section{A. Simulation set-up}

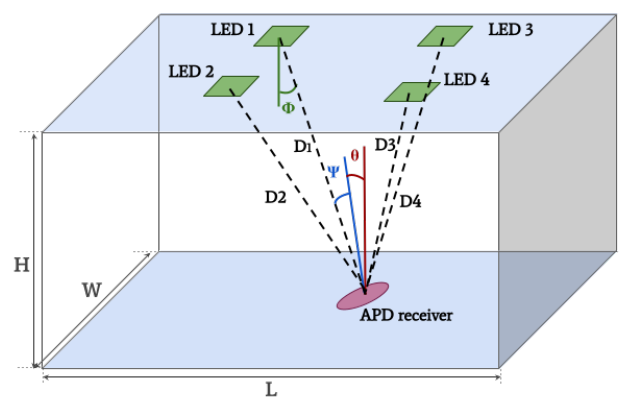

Fig. 3. A typical indoor VLP scenario

A typical indoor VLP scenario was established with the room size of $5 \mathrm{~m} \times 5 \mathrm{~m} \times 3 \mathrm{~m}$, which is illustrated in Fig. 3 .

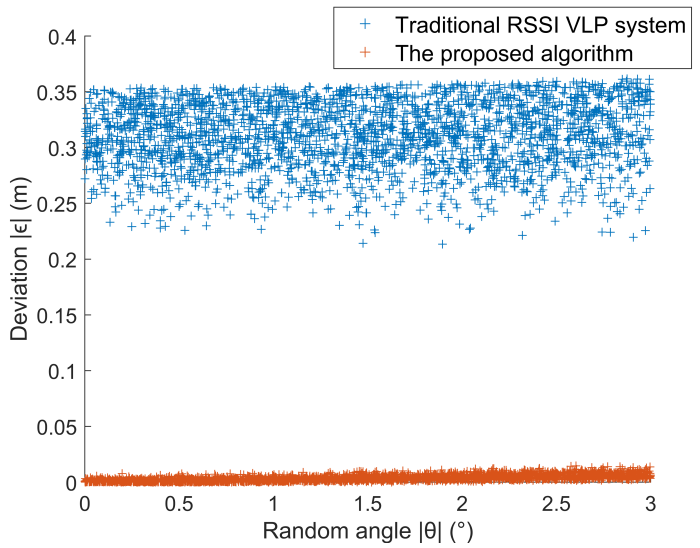

Fig. 4. Deviation of our proposal and traditional RSSI VLP algorithm

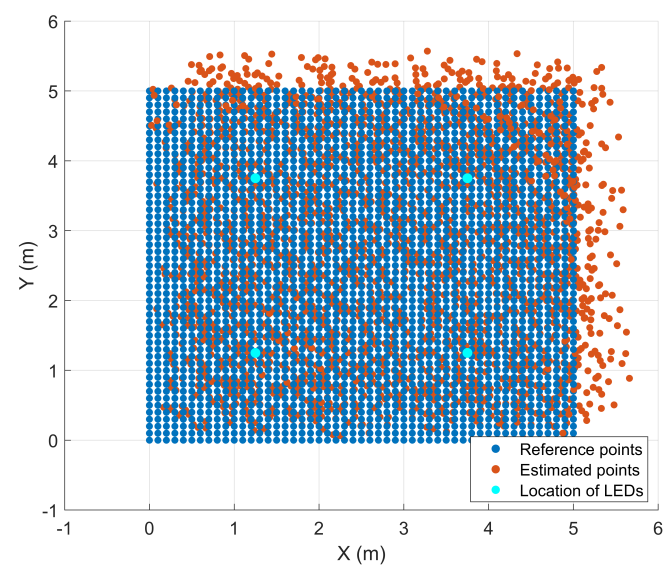

Fig. 5. Positioning results using traditional RSSI based VLP system

Four LEDs were deployed on the ceiling downward vertically . The receiver was posed on the floor at the height of 0.85 m. Totally 2601 reference points were evenly distributed in positioning area. Signals from transmitters in Line-of-Sight (LOS) propagation with Gaussian white noise were generated. During the signals collection, receiver tilted a random angle $\theta_{i}, \theta \in\left[-3^{\circ}, 3^{\circ}\right]$ at each point. The simulation parameters are detailed in Tab. I.

\section{B. Results and Analysis}

Firstly, the comparison between our proposal and traditional RSSI VLP algorithm on deviation $\epsilon$ is shown in Fig. 4. It is obvious that our proposal gained less deviation than the traditional approach. The maximum and minimum deviation declined from $0.36 \mathrm{~m}$ to $0.01 \mathrm{~m}$, and from $0.2133 \mathrm{~m}$ to $1.33 \times 10^{-5} \mathrm{~m}$, respectively. Meanwhile, the average deviation came to $3.5 \times 10^{-3} \mathrm{~m}$ from $0.31 \mathrm{~m}$.

Positioning results of traditional RSSI based VLP algorithm and the proposed algorithm are shown in Fig. 5 and 6. In Fig. 5 , it is obvious that the reference points are mostly far from the real position. The minimum and maximum PE are 0.0008 $\mathrm{m}$ and $1.3676 \mathrm{~m}$, and the average PE is $0.4633 \mathrm{~m}$. Fig. 6 shows 
TABLE I

INDOOR VLP SYSTEM SIMULATION PARAMETERS

\begin{tabular}{|c|c|}
\hline Parameters & Typical values \\
\hline Position area $(\mathrm{m} \times \mathrm{m} \times \mathrm{m})$ & $5 \times 5 \times 3$ \\
\hline Coordinates of transmitters $(\mathrm{m})$ & $(1.25,1.25,3)(1,25,3.75,3)(3.75,1.25,3)(3.75,3.75,3)$ \\
\hline Height of transmitters $(\mathrm{m})$ & 3 \\
\hline Height of receiver $(\mathrm{m})$ & 0.85 \\
\hline Number of reference points of receiver & 2601 \\
\hline Angle of receiver $\left(^{\circ}\right)$ & Random in $[-3,3]$ \\
\hline Transmitted power of LED $(\mathrm{W})^{\circ}$ & 25 \\
\hline Effective area of receiver $\left(m^{2}\right)$ & $1 \mathrm{e}-4$ \\
\hline
\end{tabular}

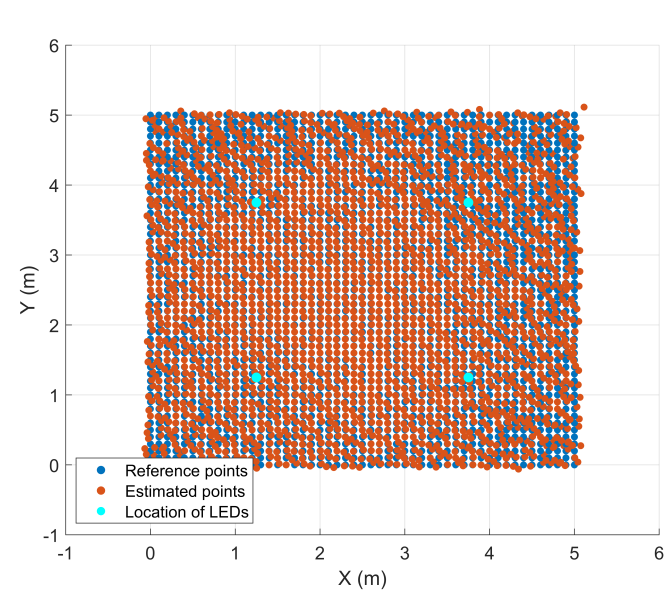

Fig. 6. Positioning results using the proposed algorithm

our proposal that reference points and estimated points almost overlapped. The minimum PE can achieve $0.0001 \mathrm{~m}$ and the maximum PE is only $0.1603 \mathrm{~m}$ and the average PE comes to $0.0247 \mathrm{~m}$.

According to the results, by employing the proposed algorithm, the minimum $\mathrm{PE}$ and the maximum $\mathrm{PE}$ declined by $87.5 \%$ and $88.28 \%$, meanwhile the average PE declined by $94 \%$. It indicates that our proposal mainly eliminates the interference of random angle.

\section{EXPERIMENTAL DEMONSTRATION}

In this section, an experimental demonstration was employed to validate the performance of proposed algorithm in a realistic scenario. The platform of this demonstration is based on $5 \mathrm{G}$ Internet of Radio Light (IoRL) which belongs to the European project Horizon 2020 [15]. Among the demonstration, real 5G NR signals were generated and utilized as PRSs to estimate the performance of our proposal. The demonstration setup and the results are detailed as follows.

\section{A. Demonstration Setup}

This demonstration was employed on a 5G NR signal based VLP system. A flow chart of the experiment and its realistic scenario are shown in Fig. 7. Four LEDs were fixed on the ceiling with a known location. The receiver kept $1.042 \mathrm{~m}$ height and moved on a plane parallel to the ground. During the movement, the tester deliberately inclined the receiver with a random angle which was within $\left[-5^{\circ}, 5^{\circ}\right]$.

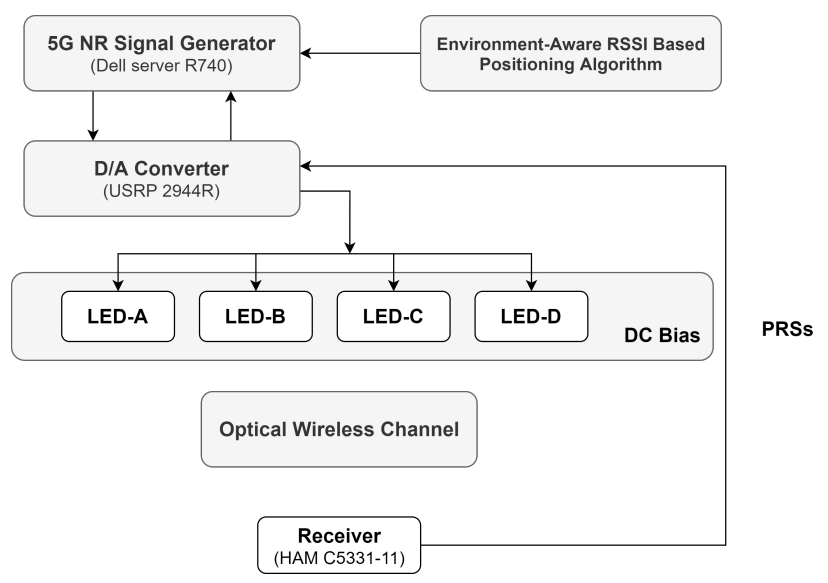

(a)

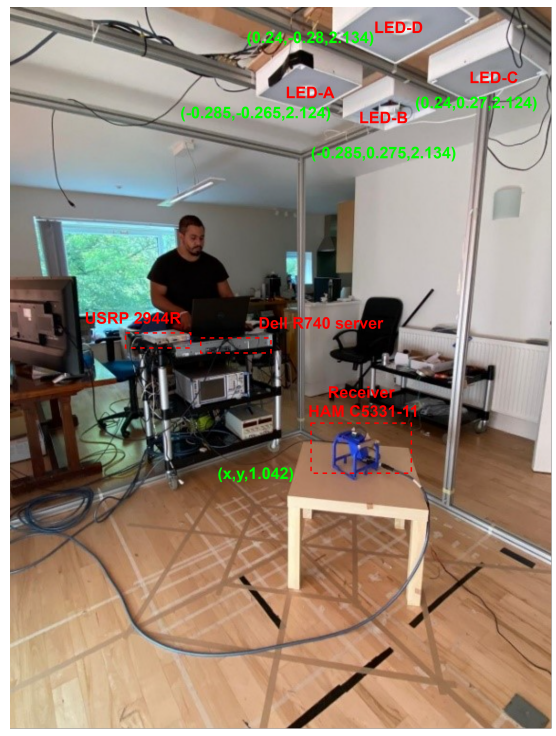

(b)

Fig. 7. (a )A flow chart of the demonstration, (b) Realistic scenario

A server (Dell R740) acted as the 5G NR baseband signal generator modulates the user data with different modulation schemes which included QPSK, 16-QAM, 64-QAM and 256QAM. As the modulated symbols were assigned to the $5 \mathrm{G}$ NR frame, as shown in Fig. 8, Inverse Fast Fourier Transform 


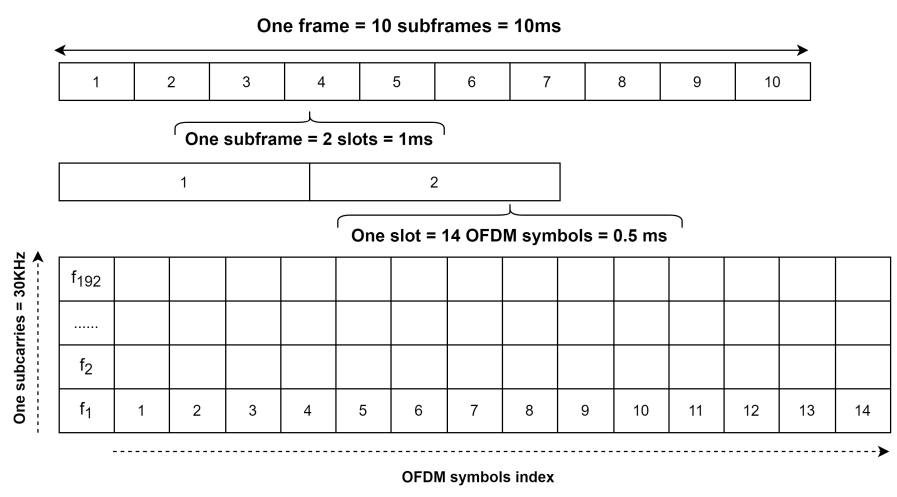

Fig. 8. Structure of 5G-NR signal among the demonstration

(IFFT) processing and IQ modulation were applied to convert the OFDM frame to time-domain and guarantee the signal well matched with the Intensity Modulation / Direct Detection (IM/DD) VLC system. Subsequently, a digital-analog (D/A) converter (USRP 2944R) and a DC bias transformed this digital signal to analog signal with an appropriate DC component. This signal is sent to four LEDs (LUXEN 5050). Through an OWC, the signal from each LED is captured by a commercial receiver (HAM C5331-11) in sequence and digitized by analog-digital (A/D) converter (USRP 2944R). Signal samples from each LED were collected 30 times by the receiver at 24 different points. Using the proposed algorithm, 96 environment indexes were extracted from all the samples. These environment indexes and signal power of the all samples were put into a multi-SVM classifier to train the mapping which introduced previously as classification model.

Once the classification model had been trained, another 20 times test on each point for each LED were employed exactly in the same way. Each sample was enable to obtain an environment index which was predicted by the classification model. By using their indexes and PRSs, the estimated coordinate can be calculated by the proposed algorithm.

\section{B. Demonstration Results}

Fig. 9 and 10 show positioning results of traditional RSSI based VLP system and the proposed algorithm. The positions of four LEDs, the reference points and the estimated points are represented by yellow, blue and red color on both figure, respectively. For traditional approach, the minimum and the maximum PE were $4.10 \mathrm{~cm}$ and $43.69 \mathrm{~cm}$, and the average PE was $22.34 \mathrm{~cm}$. By employing our proposal, the minimum $\mathrm{PE}$ and maximum PE came to $0.55 \mathrm{~cm}$ and 20.34, and the average was $5.97 \mathrm{~cm}$. Additionally, Fig. 11 and Fig. 12 show Cumulative Distribution Function (CDF) results of traditional RSSI based VLP system and the proposed algorithm. Here, 10 $\mathrm{cm}$ is considered as an acceptable PE, and only $48 \%$ results of the traditional VLP system meet the requirement, fortunately, our proposal increased it to $78 \%$.

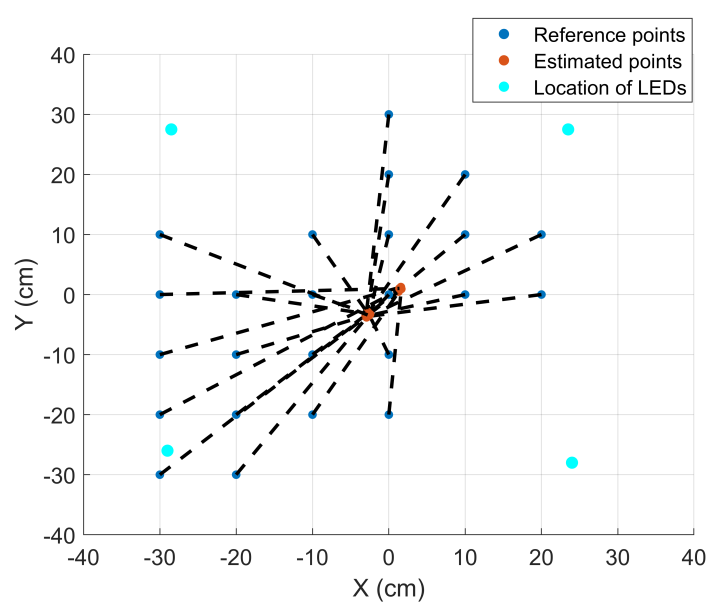

Fig. 9. Demonstration results using traditional RSSI based VLP algorithm

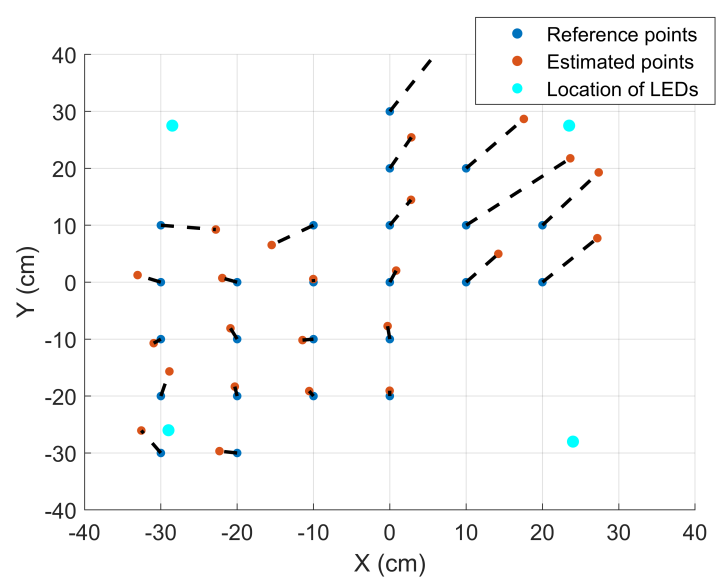

Fig. 10. Demonstration results using the proposed algorithm

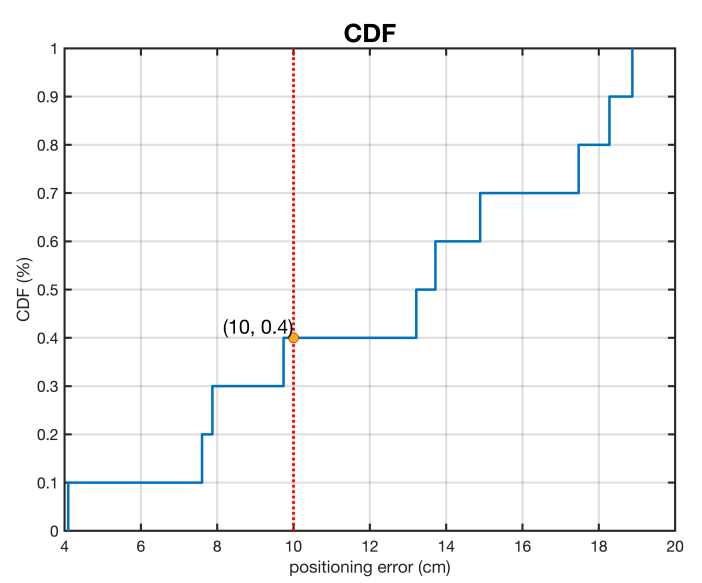

Fig. 11. Cumulative Distribution Function(CDF) of traditional RSSI based VLP system 


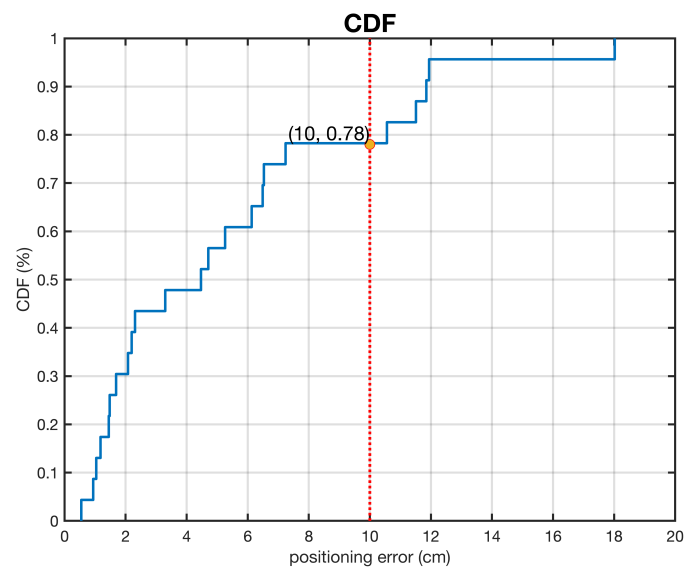

Fig. 12. Cumulative Distribution Function(CDF) using the proposed algorithm

\section{CONCLUSION AND Discussion}

In this paper, a novel algorithm is proposed to eliminate the interference of random angle deviation. The impact of this deviation on positioning accuracy was theoretically analyzed and validated by numerical simulation. According to the simulation results, our proposal effectively inhibits the random angle deviation. The average Positioning Error (PE) declined by $94 \%$. Additionally, a demonstration on a laboratory platform with 5G NR signals was implemented. The average PE declined by $73.28 \%$. In this paper, to focus on the interference of random angle, we assumed that the light propagation is Line-of-Sight. the Non-Line-of-Sight propagation will be considered in the future investigation. In addition, different kinds of machinelearning based classifier will be compared and implemented to further increase the performance of our algorithm.

\section{REFERENCES}

[1] P. Enge and P. Misra, "Special issue on gps: The global positioning system," Proceedings of the IEEE, pp. 3-172, 1999.

[2] J. Rojo, G. M. Mendoza-Silva, G. Ristow Cidral, J. Laiapea, G. Parrello, A. Simó, L. Stupin, D. Minican, M. Farrés, C. Corvalán, F. Unger, S. M. López, I. Soteras, D. C. Bravo, and J. Torres-Sospedra, "Machine learning applied to wi-fi fingerprinting: The experiences of the ubiqum challenge," in 2019 International Conference on Indoor Positioning and Indoor Navigation (IPIN), pp. 1-8, 2019.

[3] A. Sato, M. Nakajima, and N. Kohtake, "Rapid ble beacon localization with range-only ekf-slam using beacon interval constraint," in 2019 International Conference on Indoor Positioning and Indoor Navigation (IPIN), pp. 1-8, 2019.

[4] H. Liu, H. Darabi, P. Banerjee, and J. Liu, "Survey of wireless indoor positioning techniques and systems," IEEE Transactions on Systems, Man, and Cybernetics, Part C (Applications and Reviews), vol. 37, no. 6, pp. 1067-1080, 2007.

[5] Y. Gu, A. Lo, and I. Niemegeers, "A survey of indoor positioning systems for wireless personal networks," IEEE Communications surveys \& tutorials, vol. 11, no. 1, pp. 13-32, 2009.

[6] X. P. Li, L. Chen, and M. Chen, "An approach of LED lamp system lifetime prediction," in 2011 IEEE International Conference on Quality and Reliability, ICQR 2011, pp. 110-114, 2011.

[7] C. C. Lee, C. S. Tan, H. Y. Wong, and M. B. Yahya, "Performance evaluation of hybrid VLC using device cost and power over data throughput criteria," in Ultrafast Imaging and Spectroscopy, vol. 8845, p. 88451A, SPIE, sep 2013.
[8] Z. Zhou, M. Kavehrad, and P. Deng, "Indoor positioning algorithm using light-emitting diode visible light communications," Optical engineering, vol. 51, no. 8, p. 085009, 2012.

[9] S. Li, S. Shen, and H. Steendam, "A positioning algorithm for vlp in the presence of orientation uncertainty," Signal Processing, vol. 160, pp. 13-20, 2019.

[10] E. M. Jeong, S. H. Yang, H. S. Kim, and S. K. Han, "Tilted receiver angle error compensated indoor positioning system based on visible light communication," Electronics Letters, vol. 49, pp. 888-890, jul 2013.

[11] S. Li, S. Shen, and H. Steendam, "A positioning algorithm for VLP in the presence of orientation uncertainty," Signal Processing, vol. 160, pp. 13-20, jul 2019.

[12] D. H. Mai, H. D. Le, T. V. Pham, and A. T. Pham, "Design and Performance Evaluation of Large-Scale VLC-Based Indoor Positioning Systems under Impact of Receiver Orientation," IEEE Access, vol. 8, pp. 61891-61904, 2020.

[13] Z. Ghassemlooy, W. Popoola, and S. Rajbhandari, Optical wireless communications: system and channel modelling with Matlab®. CRC press, 2019.

[14] Y. Liu and Y. F. Zheng, "One-against-all multi-class SVM classification using reliability measures," in Proceedings of the International Joint Conference on Neural Networks, vol. 2, pp. 849-854, 2005.

[15] L. Shi, D. Shi, X. Zhang, B. Meunier, H. Zhang, Z. Wang, A. Vladimirescu, W. Li, Y. Zhang, J. Cosmas, K. Ali, N. Jawad, R. Zetik, E. Legale, M. Satta, J. Wang, and J. Song, "5G Internet of Radio Light Positioning System for Indoor Broadcasting Service," in IEEE Transactions on Broadcasting, vol. 66, pp. 534-544, Institute of Electrical and Electronics Engineers Inc., jun 2020. 